\title{
METAINVESTIGACIÓN DE LA FORMACIÓN, UNA PERSPECTIVA DESDE LOS ESTUDIOS REGIONALES PARA LA COMPRENSIÓN DE LAS RELACIONES DE FORMACIÓN
}

\author{
Dra. Diana Lizbeth Ruiz Rincón \\ UNIVERSIDAD AUTÓNOMA DE CHIAPAS \\ dianaruizrincon@gmail.com
}

\section{INTRODUCCIÓN}

El siguiente texto tiene por objeto presentar una serie de reflexiones en torno a la noción de epistemología de la formación, como una perspectiva planteada desde los estudios regionales para la comprensión de las relaciones de formación en la formación de investigadores.

Teniendo presente que nuestra herencia más próxima, como hijos de la modernidad, poseemos un caudal de modelos teóricos que manan de una racionalidad positiva y lineal, donde la realidad es desvelada, des-cubierta por sujetos distantes a ella. Con una fuerte presencia dicotómica entre el sujeto que conoce y el objeto cuya única cualidad (epistémica) consiste en ser aprendido y aprehendido por el sujeto pensante.

Bajo esta perspectiva operacionalizamos nuestras creencias e inclusive nuestras certezas. El conocimiento se encuentra jerarquizado, partiendo del 
entendimiento de la realidad como una estructura, nos señalan Deleuze y Guattari (2014), el conocimiento mismo se organiza de manera vertical, donde en la base más fuerte (haciendo uso de la metáfora arbórea) son las ciencias duras, las más abstractas, las que proveen de leyes generales y primeros principios a las ciencias de segundo orden, como las ciencias sociales. Esta organización vertical, utilizada también por Marx para representar la estructura (sub y superestructura) social, es la visión con la que estos autores presentan, mediante conceptos como el de rizoma, buscan plantear -o plantar- un conato de resistencia. Desplegando así otra forma de verse en la realidad (nótese la omisión de la sentencia "observar la realidad", que al enunciarse contiene ya una distancia epistemológica pero también ontológica entre el sujeto y el objeto).

Por un lado niegan la necesidad de que los contenidos de cualquier saber deba configurarse según ese modelo de orden arborescente, "la metáfora del rizoma (...), posibilita modificar radicalmente la concepción básica de la epistemología tradicional (desde el platonismo y aristotelismo a la misma filosofía analítica) al prescindir de la diferencia básica entre sujeto y objeto" (Deleuze \& Guattari, 2014, pág. 12). De esta manera, “(...) lo rizomático o los rizomas carecen de unidad que pueda ser centrada, se establecen relaciones y conexiones transversales. No existen puntos en el rizoma y sí líneas interconectadas en procesos continuos y cambiantes" (Deleuze \& Guattari, 2014, pág. 8).

Los entes del mundo (sujetos, objetos, objetos subjetivados, sujetos objetivados, sujetos sujetados a objetos, etc.), se encuentran inmersos en una 
estructura, en múltiples estructuras, y se encuentran en un constante siendo, en un constante movimiento o cambio. El hombre despliega sus capacidades y posibilidades, sus actos y sus potencias de manera permanente, inmerso en un sistema, en una estructura sistémica que va transformándose a la par de las modificaciones de las relaciones que se suscitan y suceden diacrónicamente (y anacrónicamente -porqué no-).

El sujeto que va configurándose a partir de sus relaciones con el entorno, con la otredad, la alteridad, los entes, se configura y reconfigura la estructura en la que participa, es algo más allá de lo dado, es lo siendo. Como apelar a la resistencia de una verdad absoluta, más bien a una constante configuración, ruptura, resistencia, continuidad, discontinuidades, etc.

Por último, en lo que respecta a los planteamientos estructuralistas, la noción de formación que se recupera tanto de Honoré (1980), como de Ferry (1990), y tienen que ver, en el caso del primero, con entender la formación como actividad estructurada a partir de marcos de diferenciación -en función de distintos modos de formación que tienen cabida dentro de la formatividad- para ejercer disposiciones y desplegar potencialidades evolutivas, con la presencia de recorridos procesuales de diferenciación e integración, entre otras antinomias. Por su parte, Ferry ve a la formación como un trayecto -continuo y discontinuo- que tiene lugar dentro de una institución, entendiendo a ésta como un dispositivo organizacional que estructura las experiencias y prácticas formativas y formadoras. 
Pero luego de estas acotaciones, ¿cómo entenderemos a la formación a partir de los elementos antes expuestos?

\section{DESARROLLO}

Bernard Honoré (1980), para quien la formación es un proceso que permitirá al hombre no solo conocer, sino elegir las finalidades en términos de aplicación de dicho conocimiento, define la formatividad como un campo que se constituye a partir de un conjunto de fenómenos relacionales de transformación en un determinado tiempo, así como de su organización.

La actividad formativa como función de la misma formación comprende la experiencia de este proceso, pero no se reduce al mero aprendizaje que, es generalmente considerado como una relación en la que un sujeto pasivo recibe de otro ciertos contenidos. Dicha actividad formativa trasciende la pasividad del aprendizaje, vinculando activamente al sujeto y sus experiencias en distintos niveles de la vida y su pensamiento.

La propuesta de Honoré, en correspondencia con la perspectiva que plantea el estructuralismo, invita a la eliminación de la dicotomía sujeto-objeto, en su caso 
la eliminación de la dualidad formante-formado. Lo anterior no significa que no deban existir fronteras en marcos conceptuales, sino que en el acaecer diacrónico de los hechos -educativos- de la realidad pueda comprenderse en términos de relaciones. (2009, pág. 85)

En la formatividad como acción, el sujeto formante se forma como parte del mismo proceso de formación. Esto es, participante de la estructura de la realidad, el sujeto -que no es distinto ni ocupa un lugar central en el campo del conocimiento, o de lo que es cognoscible-, al actuar en formatividad transforma su entorno y a sí mismo, formando y siendo formado en este intercambio energético (entrópico) con el mundo que lo conforma y del que a su vez recibe y da forma.

A primera vista, (...) se constata que las condiciones institucionales y metodológicas de la formación profesional se organizan muchas veces de manera tal que es sobre la diferencia profesional, e incluso sobre la diferencia institucional, donde se fundan las prácticas de formación. Éstas llegan, en el seno de la profesión, o de la institución al refuerzo de lo que es idéntico, al mantenimiento de las jerarquías, a una cohesión mayor de una estructura especializada, pero encerrada en sí misma, bloqueada en sus potencialidades evolutivas, (...). (Honoré, 1980, pág. 110).

A decir de las particularidades antes citadas, en la que la formación profesional marca la diferenciación en la formatividad a partir de los marcos institucionales, disciplinares e inclusive organizacionales, Honoré define la formatividad como "el 
conjunto de los hechos que conciernen a la formación, considerada como función evolutiva del Hombre" (1980, pág. 125). Dicha función evolutiva se toca con las nociones de desarrollo, cambio o transformación, en la que como resultado de la acción formativa -que no es la única que participa en la forma en la que la perspectiva sistémica apunta que se "realiza" la realidad- habrán de suscitarse y sucederse en un espacio y tiempo determinados, transformaciones como resultado del proceso de formación.

La formatividad es el campo de la mirada y de la acción sobre la evolución. En este campo encontraremos, pues, un cierto número de hechos que provienen de la experiencia:

- todo lo que caracteriza la actividad reflexiva sobre los fenómenos evolutivos;

- todo lo que caracteriza la actividad de formación donde encontramos lo que «es puesto en marcha», concerniente a la evolución, esos dos aspectos se complementan en la noción de investigación de acción a propósito de la formación;

- las condiciones de esta actividad, en el espacio y en el tiempo.

La formatividad puede también entenderse como el carácter de lo que es formativo, es decir lo que es una condición favorable, o que ejerce una disposición, un poder para favorecer el proceso de formación. Representa, pues la manera en que el entorno material y humano toma las «formas» que, 
en el medio, sirven de soporte objetivo a la formación (Honoré, 1980, pág. 126).

En el caso de la educación formal, el entorno material estará conformado por un sistema educativo estructurado en distintos niveles; en el caso del sistema educativo mexicano, son mayormente visibles los niveles en el siguiente orden: Básico, Medio Superior y Superior; organizados a partir de distintas duraciones, objetivos, normas, disposiciones en términos de infraestructura y otros sujetos formadores-, cuya función formativa, en el caso del nivel profesional, pero más específicamente en el nivel del posgrado, estará mayormente realizada en el terreno de la formación para la investigación. Al respecto Honoré incorpora las siguientes nociones para dar cuenta de las principales características de la actividad formativa estructurada:

- la actividad formativa está fundada en la experiencia relacional, vivida en un entorno histórico físico y social;

- está fundada sobre la diferenciación en el presente, del pasado y del porvenir;

- está sometida a la discontinuidad temporal y espacial de las funciones de afirmación y a las funciones de adaptación;

- se manifiesta con una particular intensidad durante los fenómenos de ruptura; 
- es una elaboración permanente de la información, desde la señal hasta la significación, con una integración de todas las etapas que jalonan el recorrido de una elaboración, cuyo proceso es la reflexión;

- se deduce de aquí que la actividad formativa es una actividad de evaluación (Honoré, 1980, págs. 138-139).

Nuestro autor construye un bosquejo teórico a partir de las relaciones que se establecen entre las nociones de relación y cambio, espacio y tiempo, así como otros términos como el de intención, significación, organización, etc. El trabajar pues, con un ejercicio teorizador de la formación, involucra:

(...) un doble proceso de diferenciación-integración de toda forma en una forma nueva, y de transformación de la energía física en vital, en psíquica, en intencional. (...) Bosquejo teórico según el cual nosotros consideramos la formación como una función que se ejerce según un proceso de diferenciación-integración y de activación significativa. (Honoré, 1980, págs. 13-14)

Por otro lado, Gilles Ferry (1990) define la formación en tres principales aspectos. Primero la entiende como una función social de trasmisión, luego como un proceso de desarrollo y estructuración de la persona; empero, la definición de formación que habrá de recuperarse de este autor, para los fines que esta investigación persigue será entender la formación como una institución, a saber: 
Una institución es un dispositivo organizacional que, por ejemplo, está hecho de programas, de planes de estudio, de certificaciones, de construcciones. Es precisamente en estos términos que siempre se ha evocado la formación dentro de la esfera de la enseñanza (...) (Ferry, 1990, pág. 51).

Ferry (1990) permite situar la formación en un marco de actuación institucional, donde lo instituido y lo instituyente configuran el escenario en el que habrá de desplegarse la formatividad que nos señala Honoré (1980).

Una institución es también el lugar de unas prácticas, normas, sus modelos, su propia tecnicidad, su lenguaje fácilmente trivializable (las demandas, los objetivos, las estrategias, la evaluación, el control) y sus practicantes, los formadores. Los formadores que desarrollan una acción en este espacio transicional y transaccional entre los conjuntos sociales y los individuos, y que se perciben ya sea como agentes de cambio social o como incitadores de deseos o de proyectos personales (Ferry, 1990, pág. 51).

El concepto de formación, desde la perspectiva estructural de la que se sirven tanto Honoré como Ferry, permite comprender esta función/acción formativa en una dimensión procesual, en donde las dinámicas involucradas y las relaciones que representan se configuran a la vez a partir de rupturas, tensiones, continuidades y discontinuidades en el accionar de las potencialidades que se buscan desarrollar. De esta manera, la formación y su trayecto en espacios y tiempos configurados a 
partir de rutas marcadas e instrumentadas permite a Ferry ubicar al ser humano en su característica social, en el que dicho proceso de formación:

(...) no se desarrolla sino a través de interacciones, de integraciones con grupos, de pertenencias a una clase, y que supone ciertos requisitos sociales: incitación, modelos, sanciones que de todas formas, aun en el caso del más libre vagabundeo cultural, aun cuando no se comprendan muy bien, orientan y estructuran el proceso. Es deseable representarse estos dispositivos o estos medios de formación como múltiples, entrecruzados, frecuentemente antinómico (contradictorio) (Ferry, 1990, pág. 54).

Empero, como bien señala Ferry, "si consideramos la transmisión de los conocimientos como el aspecto prioritario de la función enseñante, y el nivel de la calificación científica aparece como primer objetivo, la especialización se impone" (1990, pág. 56). Lo anterior implica pues, que dentro de las complejas relaciones en toda actividad formativa, la que se refiere a la formación para la generación y aplicación del conocimiento en campos disciplinarios específicos pero en encuentro, abarcará a la vez determinados dispositivos desde los cuales se moldearán los distintos modos de entender la ciencia y su realización.

Al respecto Ferry (1990) agrega que a "los investigadores o a los practicantes, con frecuencia se les solicita no sólo comunicar sus "resultados" y "experiencias", sino dar clases en relación con su especialidad" (Ferry, 1990, pág. 
58), ello tendrá diversas implicaciones en las relaciones que se susciten en el dispositivo que la institución articule, en tanto que:

Los enseñantes en formación, así como otras categorías de formadores, son sometidos a fuertes tensiones, por el hecho de que la problemática y las prácticas de formación son para ellos tanto el objeto de estudio, de reflexión, de compromiso simbólico, como vivencias cotidianas de múltiples avatares en la realidad de la institución formadora (Ferry, 1990, pág. 62).

El término "formación de investigadores", como tal, tendrá dos fuentes principales. Por un lado, Imre Lakatos (2011), desde la reconstrucción racional de la ciencia como estructuras, acuña el concepto de programas de investigación, en el cual los futuros investigadores se forman y adscriben, sin que ello implique una 72 desvinculación (inconmensurabilidad en palabras de Kuhn) entre los distintos programas y las teorías que los soportan. La formación, en estos programas de investigación científica, será lo que nos ocupará en un desarrollo posterior.

Así, desde esta caracterización de la formación, puede irse esbozando el lugar que guarda la formación de investigadores en el nivel del doctorado dentro del sistema educativo mexicano. Resulta necesario, independientemente del campo disciplinario en el que se adscriba, hacer un esfuerzo por analizar y tratar de dar cuenta de las formas en las que, dentro de este proceso formativo la formación misma es entendida. 
Puesto que se han abordado diversos puntos respecto a lo que habrá de entenderse por epistemología de la formación, entender de qué manera en cada actor está vigente aquella trayectoria ideal que regula, expresada en una pauta curricular, en términos de producción, obliga a la compartimentación de los aspectos imbricados en dicho proceso de formatividad.

Se tratará entonces de una cuestión de cada organización en relación con el proyecto formativo que la orienta y que lleva adelante en el día a día, atravesada por distintas variables de su estructura organizativa (...): los fines de la institución [dispositivo] en tanto idea de rumbo, de propósito que organiza la acción y provee sentido (...) los significados culturales propios, que andamian más o menos, que permiten mutar o neutralizar cualquier desviación de aquello del orden de los que se espera; el espacio tanto en su capacidad material como simbólica, como andamiaje y como articulador del tiempo (...). (Nicastro \& Greco, 2009, pág. 43)

Las variables y rasgos de las trayectorias presentadas a continuación, expondrán aspectos de visibilización de los posibles modos de relación, a partir de los cuales será observado el aspecto de la realidad que atañe a los dispositivos de formación de investigadores en el campo de los estudios regionales en México.

Por su parte, las variables corresponderán a líneas que atraviesan los dispositivos, tanto visibles como no visibles, y que su trazamiento podrá estar sujeto a cambios en función de los dispositivos y sus elementos caracterizadores. 
Por otro lado, los rasgos comprenderán las características, las particularidades de la acción o práctica formativa comprendida en la trayectoria.

Nicastro y Greco (2009) exponen una serie de rasgos y variables para el análisis de dispositivos de formación, los cuales serán recuperados, agregando distintos modos posibles de relación entre ellos, de manera que puedan considerarse como categorías de observación en el posterior y necesario trabajo de campo.

Las variables a identificar en relación a las sedes serán: los fines de la institución, los significados culturales, el espacio material y simbólico, y el tiempo. Cada una de estas particularidades permite observar en los dispositivos como instituciones, aspectos que les aproximan en términos de semejanza, pero también talantes que los distancian en función de aquellos sentidos que les otorgan identidad y los configuran de manera distinta, es decir, que los distinguen.

Los fines de la institución estarán caracterizados al interior de cada dispositivo, cuya formulación no necesariamente distará de otras formas instituidas. Estos fines estarán marcados como pautas y guías principalmente en los planes de estudio, en los que se establecer los modos en que los programas de estudio de los doctorados en estudios regionales entienden el campo, así como las características de la formación de investigadores en ese mismo aspecto. 
Los significados culturales serán aquellos contextos desde los cuales fueron creados y tomaron forma los distintos dispositivos en cada sede, atendiendo algunas características históricas. Por su parte, el espacio material y simbólico corresponderá a las fronteras que marcan semejanzas y diferencias entre las sedes. En este sentido, la idea de territorio que será desarrollado posteriormente, permitiría a esta investigación en su acepción regional. Finalmente, la categoría de tiempo en el análisis de los dispositivos como sedes, atenderá a la duración del programa, las implicaciones de los encuentros entre los actores noveles y expertos, y la caracterización de las prácticas rutinizadas en torno a la formación para la investigación.

Lo anterior se convierte en antesala para el entendimiento situacional y el ejercicio de una mirada focalizada en los rasgos de formación en la trayectoria, como parte de las categorías desde las cuales se realizará el trabajo de análisis de los dispositivos, que presentan condiciones de entrecruzamiento con las variables; y que pueden ser, a partir de la propuesta de Nicastro y Greco (2009): la inactualidad, la finitud, el inacabamiento y la interrupción.

Inactualidad refiere a que no sólo se forma para un aquí y un ahora que tenemos de alguna manera asegurado y hasta atrapado, sino en un espacio y en un tiempo que está fuera de nuestro control actual. (Nicastro \& Greco, 2009, pág. 49) Es más bien un proceso constante dentro y fuera de la institución, en donde es posible usar a manera de analogía la idea de constante devenir, esto es, en el que el proceso de formatividad no se concluye y cierra toda posibilidad de ejercicio 
ulterior, más bien donde es la práctica misma de formación la que trasciende el mero accionar de la formatividad y la despliega, más allá de la institución.

Desde acá la formación en su rasgo de finitud se traduce en un trayecto que tiene un tiempo en el marco de una institución, que se hace cargo allí de algunas cuestiones sin intentar abarcar todo lo que la inactualidad pone por delante como porvenir. (Nicastro \& Greco, 2009, pág. 50)

La finitud sería el tiempo/duración en la conformación de la trayectoria de formatividad, que lleva a la idea de inacabamiento. Entendiendo al proceso de formatividad como una dinámica inacabada, donde es necesario no sólo una programación, sino también una reprogramación, a partir de la última categoría dentro de los rasgos de la formación en la trayectoria, el de interrupción, como un despliegue para la reflexión y el cuestionamiento atendiendo a la producción. De alguna manera la formación adquiere la cualidad de intervalo cuando se interrumpe el proceso, el progreso, el retroceso, como si el tiempo dejara de ser un tiempo cronológico y continuo, orientado hacia un solo lugar. (Nicastro \& Greco, 2009, pág. 52)

Si bien en las estructuras sistémicas hay un elemento regulador externo, están presentes elementos comunes pero también variabilidad (ruptura e individualidad), donde también emergen (comprensible dentro de la lógica del propio programa, lo dándose) otros. Por último, El proceso formativo en su dimensión común, (dentro del dispositivo/institución), lo común será el campo 
disciplinar, es decir, el campo de los estudios regionales y las fronteras cambiantes/difusas que vendrían a configurar cada dispositivo o sede.

A lo largo del primer apartado se ha abordado la noción de formación, desde una perspectiva estructural sistémica y funcional, buscando una comprensión que permita ver a la formación como una serie de elementos que se vinculan de forma no lineal, y que a la vez vehiculan la integración del aspecto institucional entre lo instituido y lo instituyente, en el cual el proceso discontinuo formativo tiene lugar dentro de determinados marcos desde donde operan y actúan los actores, así como los mecanismos, instrumentos y vías de formación que se despliegan en el dispositivo.

La noción de dispositivo, presente en la producción de Foucault liga elementos aparentemente dispersos o lejanos, y su análisis permite visibilizar dimensiones cuya implicación enunciativa muestra la manera en que tienen lugar los procesos que en este caso estarán referidos a la formación.

Deleuze (2007) retrata el dispositivo foucaultiano como:

[...] compuesto por líneas de diferente naturaleza y esas líneas del dispositivo no abarcan ni rodean sistemas cada uno de los cuales sería homogéneo por su cuenta (el objeto, el sujeto, el lenguaje), sino que siguen 
direcciones diferentes, forman procesos siempre en desequilibrio y esas líneas tanto se acercan unas a otras como se alejan unas de otras. Cada línea está quebrada y sometida a variaciones de dirección (bifurcada, ahorquillada) sometida a derivaciones. (Deleuze, 1989, pág. 155)

Este conjunto multilineal obliga a entender no al dispositivo, sino a los dispositivos presentes en el sistema que toca lo educativo. De esta manera, al interior del dispositivo en vías de caracterización debe tenerse presente que al ser un proceso no lineal estructurado dentro de un sistema de amplitud más compleja, las relaciones presentes habrán de realizarse a partir de cortes que posibiliten la enunciación y visibilidad de elementos que en su decurso se tocan, se unen, se dividen y alejan sin que ello implique la aplicación de juicios de valor con un sentido maniqueo. Esto es, teniendo como punto común la noción de formación, cada forma instituida de formatividad habrá de desplegar modos distintos de operacionalización.

Los distintos modos de expresarse en el dispositivo de formación abre espacios de ruptura que no deben ser atendidos a manera de quiebres irreconciliables o negativas, es decir, apelando a la noción de bifurcación dentro de la propuesta sistémica de Bertalanffy (1976), esta idea enuncia rutas diversas y puntos en común. Así pues,

[...] no hay una bifurcación de la razón; lo que ocurre es que ella no cesa de bifurcarse y hay tantas bifurcaciones y ramificaciones como instauraciones, 
tantos derrumbes como construcciones, según los cortes practicados por los dispositivos, y "no hay ningún sentido en la proposición según la cual la razón es un largo relato que ha terminado ahora". (Deleuze, 1989, pág. 158)

El dispositivo de formación, como algo que ha sido instituido (en la institución) de manera permanente e irrevocable, cuyo proceso conformado y estructurado ha sido concluido, elimina la posibilidad de comprender las implicaciones de sus relaciones que desde estos distintos derrumbes, bifurcaciones y caos producen contenidos y conocimiento que puede comprenderse en la medida en que los distintos niveles en las diferentes líneas que se trazan y entrecruzan en el dispositivo son analizadas.

Si bien Foucault habla de tres grandes instancias dentro del dispositivo, a saber: Saber, Poder y Subjetividad, bastará el ejercicio del primero para la presente investigación. El dispositivo de formación será comprendido inicialmente como una red articular en la que se inscriben diversas dimensiones como resultado del cruzamiento entre relaciones que pueden ser caracterizadas de acuerdo al conjunto de elementos presentes en la región a analizar, de la cual hablaremos en el siguiente capítulo.

Se trata de nombrar algunas características que conformaran y darán estructura al dispositivo de formación, como: la institución, lo instituido y lo instituyente, la trayectoria, los mecanismos, instrumentos y vías de formatividad. 


\section{ARTÍ́CULOS}

A razón de ello, una aproximación a estos dispositivos de formación considerando en primera instancia los elementos que lo hacen visible e invisible.

Hablamos entonces del dispositivo de formación como el espacio-tiempo a analizar a partir de:

a. Aspectos iniciales, recuperando de la noción de formación planteada, lo tocante a lo instituido, lo instituyente y el marco institucional.

b. Haciendo uso de conceptos como el de rizoma, fractal, bifurcación, atractor, etc.

\section{ESQUEMA № 1}

"Niveles de despliegue en la líneas de visibilidad del dispositivo de formación de investigadores"

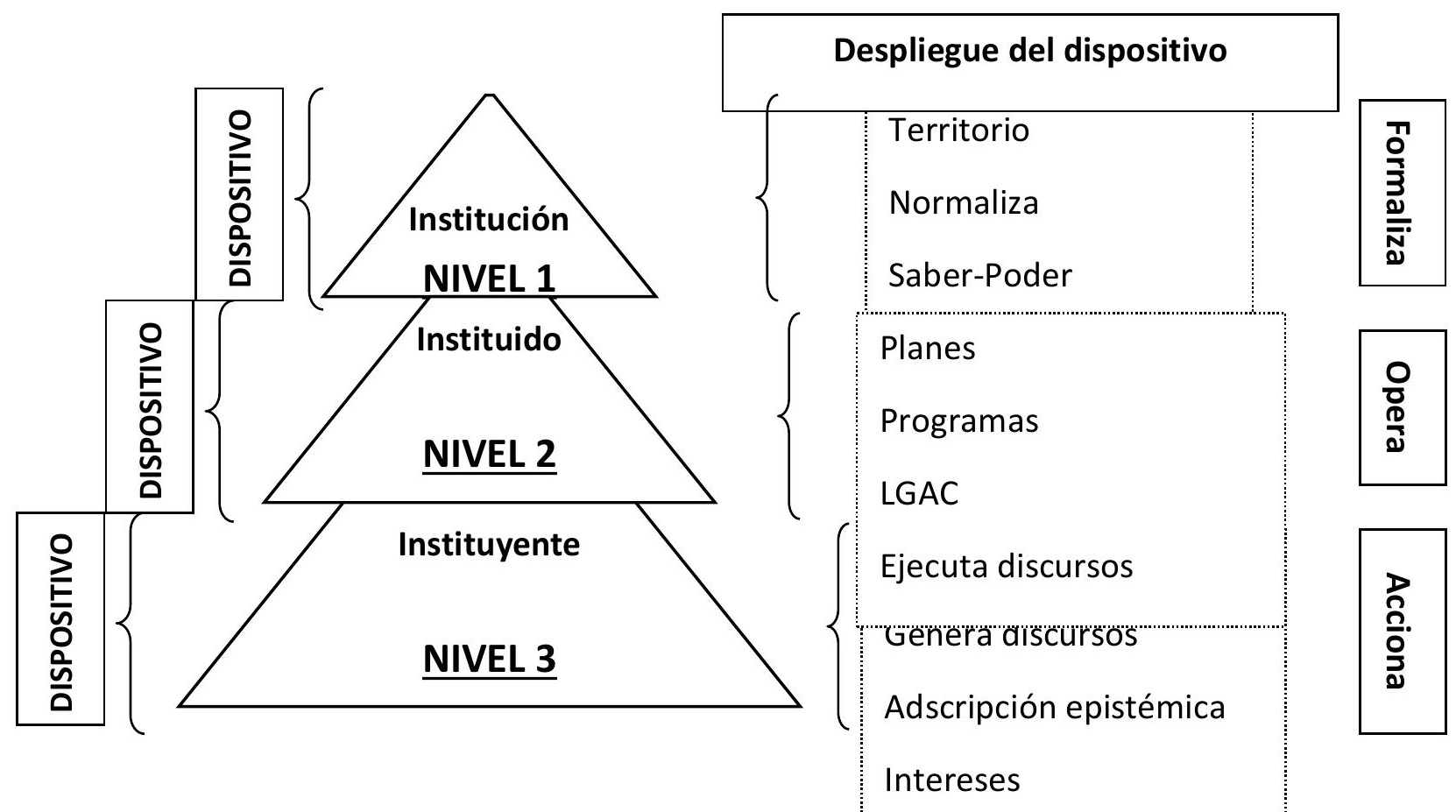




\section{ARTÍCULOS}

\section{ESQUEMA № 2}

"Estructura de líneas en relación a su visibilidad"
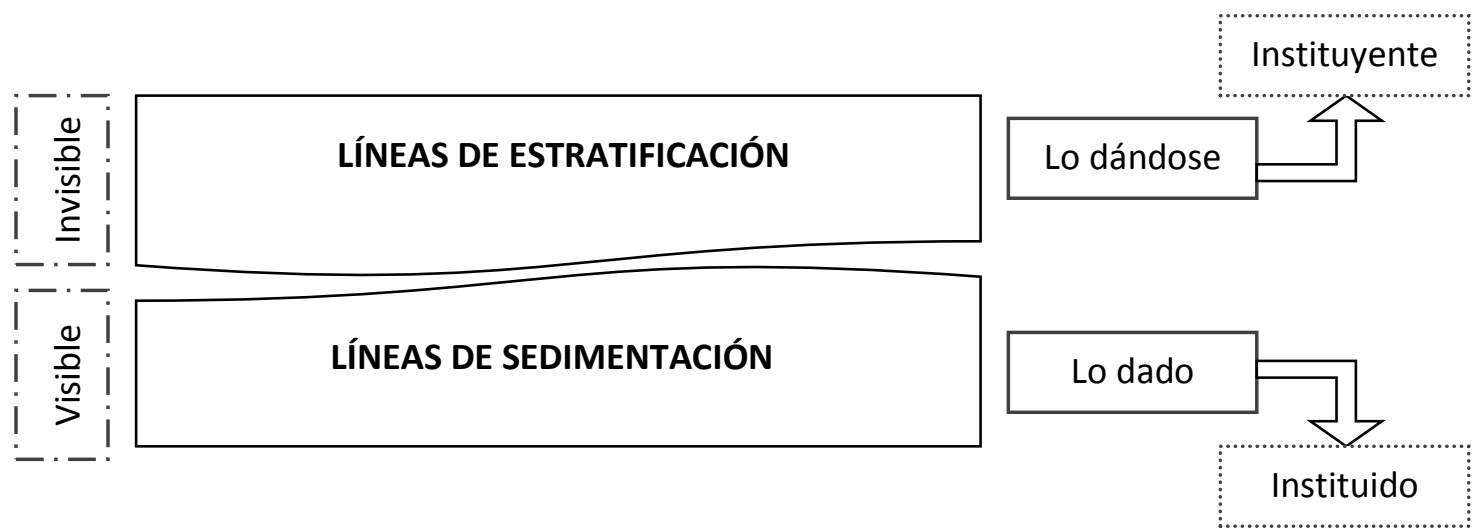

\section{$\underline{\text { REFERENCIAS }}$}

- Bertalanffy, L. v. (1976). Teoría general de los sistema: fundamentos, desarrollo, aplicaciones (Edición conmemorativa 70 aniversario, 2006 ed.). (J. Almela, Trad.) México: FCE.

- Deleuze, G. (1989). ¿Qué es un dispositivo? En E. Balbier, G. Deleuze, H. L. Dreyfus, M. Frank, A. Glücksmann, G. Lebrun, ... F. Wahl, Michel Foucault Filósofo (págs. 155-163). Barcelona: Gedisa. 
- Deleuze, G. (2007). Foucault. Barcelona: Paidós.

- Deleuze, G., \& Guattari, F. (2014). Rizoma (2ª ed.). (D. A. Rincón, Trad.) México: Fontarama.

- Ferry, G. (1990). El trayecto de la formación. Los enseñantes entre la teoría y la práctica. México: Paidós.

- Foucault, M. (2009). El orden del discurso (Fábula ed.). México: Tusquets.

- Honoré, B. (1980). Para una teoría de la formación. Madrid: Narcea, Ediciones.

- Kuhn, T. S. (2013). La estructura de las revoluciones científicas (Cuarta edición en español ed., Vol. Coles. Breviarios 213). (C. S. Peña, Trans.) México, México: Fondo de Cultura Económica.

- Nicastro, S., \& Greco, M. B. (2009). Entre trayectorias. Escenas y pensamientos en espacios de formación. Rosario: Homo Sapiens Ediciones.

- Olivé, L. (Ed.). (1995). Racionalidad epistémica (Vol. 9). Madrid, España: Trotta.

- Pérez Tamayo, R. (2003). ¿Existe el método científico?: historia y realidad. México: Fondo de Cultura Económica, SEP, CONACYT y ENC.

- Villoro, L. (2009). Creer, saber y conocer. México: Siglo XXI Editores. 